\title{
A Second-Order Finite Volume Method that Reduces Numerical Shockwave Anomalies in One Dimension
}

\author{
Daniel W. Zaide* \\ Department of Mechanical Engineering, University of British Columbia, Vancouver, Canada \\ Philip L. Roe ${ }^{\dagger}$ \\ Department of Aerospace Engineering, University of Michigan, Ann Arbor, MI, USA
}

\begin{abstract}
Numerical shockwave anomalies for the Euler equations are known to be directly related to the nonlinearity of the jump conditions and ambiguity of sub-cell shock position. To alleviate this behavior, two new flux functions were developed that permit stationary shocks with one intermediate state and an unambiguous stationary shock structure. These functions were demonstrated within a first-order framework in one dimension and shown to dramatically reduce numerical shockwave anomalies with no additional intermediate shock states or smearing. Unfortunately, four states are required for each flux function. In this work, both new flux functions are integrated into a second-order finite-volume scheme, utilizing the same four states used for reducing numerical shockwave anomalies to improve accuracy in smooth regions. Results are shown for several canonical problems and the method is shown to perform well.
\end{abstract}

\section{Introduction}

Although generally modeled as discontinuities, true shockwaves have finite width over which the energy is transformed from kinetic energy into heat, a process described by viscous dissipation. For numerical shockwaves, there is a numerical width, often much greater than the physical width. While the numerical width could be reduced to the order of the physical width, in practice this is computationally infeasible and furthermore, the governing equations do not apply at those small scales. To reconcile this disparity in length scales, two classes of schemes were developed - shock fitting and shock capturing.

Shock fitting schemes underestimate the width of the shock, treating it as an explicit discontinuity satisfying internal boundary conditions by fitting it with a cell boundary. ${ }^{1}$ This works well when the exact location of a shock is known, but is much more challenging when shocks interact and there is a complex shock structure. Shock capturing schemes, on the other hand, overestimate the width of the shock, requiring the creation of intermediate states in the shock, having no direct physical interpretation. Despite the common usage and generally good performance of shock capturing, there are still pervasive errors that can occur as a result of these intermediate states that do not disappear with common techniques such as increased grid resolution or accuracy.

These errors include errors in shock position, ${ }^{2,3}$ spurious waves, ${ }^{4-6}$ or unstable shock behavior. ${ }^{7-9}$ As they share similarities, they are classified together as numerical shockwave anomalies, numerical artifacts formed due to the presence of captured shockwaves within the flow solution. It was previously shown that each numerical shockwave anomaly is directly related to the nonlinearity of the jump conditions and to a resulting ambiguity in sub-cell shock position in a stationary shock. ${ }^{3}$

As a result of this work, two new flux functions were developed that do not have this ambiguity. ${ }^{10}$ On all of the shock anomalies in one dimension, both flux functions showed improvement on existing methods without smearing or diffusing the shock. While they are still susceptible to many of the problems that occur in Roe's Riemann solver and several other known issues, these methods served to validate the philosophy and

*Post-Doctoral Research Associate, Member AIAA

$\dagger$ Professor, AIAA Fellow 
approach: that by enforcing a linear shock structure and unambiguous sub-cell shock position, numerical shockwave anomalies are dramatically reduced.

Unfortunately, information from four cells is needed in these flux functions rather than from two cells as in a traditional Riemann solver. Introducing these new flux functions into existing second-order and higher-order methodologies is no longer trivial. This work investigates the implementation of these fluxes and the choice of cell information within the new flux functions, as increased information, such as gradients, are available. With a suitable implementation, both new flux functions are integrated into a MUSCL-type framework with increased accuracy in smooth regions of the flow and reduced anomalous behavior around shockwaves.

\section{Euler Equations}

The main physical model in this work is the Euler equations for inviscid, compressible flow. They serve as the basis for the numerical experiments performed in this work, though both new flux functions generalize to other similar symmetrizable, hyperbolic systems of equations. The Euler equations represent the conservation of mass, momentum, and energy in vector form as

$$
\mathbf{u}_{t}+\mathbf{f}_{x}=\mathbf{0}
$$

and expanded as

$$
\frac{\partial}{\partial t}\left[\begin{array}{c}
\rho \\
\rho u \\
E
\end{array}\right]+\frac{\partial}{\partial x}\left[\begin{array}{c}
\rho u \\
\rho u^{2}+p \\
\rho u H
\end{array}\right]=\mathbf{0}
$$

where enthalpy $H=\frac{E+p}{\rho}$. It is crucial to the understanding of shockwave anomalies that this system is not closed until the pressure has been defined through an equation of state. For an ideal gas in thermodynamic equilibrium, the equation of state is

$$
p=(\gamma-1) \rho e=(\gamma-1)\left(E-\frac{1}{2} \rho u^{2}\right)
$$

where $\gamma$ is the polytropic constant, the ratio of specific heats. While the ideal gas law is often used to solve problems with shockwaves, it does not apply within a shock, but rather outside the shock, with the Rankine-Hugoniot jump conditions describing the relationship between quantities on each side.

\section{Capturing Shocks}

Using a first-order in space and time finite-volume method with Roe's Riemann solver, the structure of captured shockwaves is analyzed. To represent a shockwave discretely, finite-volume methods average, or capture, shocks over several cells. These captured shocks contain intermediate states that are are not part of the exact solution but are required for the discrete representation. While the number of required intermediate states varies from scheme to scheme and depends on the Courant number and Riemann solver, ${ }^{4,11}$ among other things, all conservative schemes must produce them and they do not disappear with grid refinement.

These intermediate states are not connected to the shock end states by a single shock; instead, they are connected in part by waves of other families. As these intermediate states change, waves of all families are created, and shed around the shock. These waves are largely responsible for the shock capturing anomalies described later in this work. In fact, the aptly named "start-up error" is a manifestation of the largest waves from this - in the first steps of simulations, the large jumps between shock states result in the strongest waves shed.

The analysis focuses on the apparently trivial case of a stationary shock in one dimension. It is well-known that for both the exact and Roe's Riemann solver, a stationary shock with one intermediate state exists with solutions of the discrete equations of the form $\mathbf{u}_{L}, \ldots \mathbf{u}_{L}, \mathbf{u}_{M}, \mathbf{u}_{R}, \ldots \mathbf{u}_{R}$ for a one-parameter family of states $\mathbf{u}_{M}$. The condition that determines these states is that the fluxes computed at the interfaces between $\mathbf{u}_{L}$ and $\mathbf{u}_{M}$, and between $\mathbf{u}_{M}$ and $\mathbf{u}_{R}$ must equal $\mathbf{f}\left(\mathbf{u}_{L}\right)$ (and also, of course $\mathbf{f}\left(\mathbf{u}_{R}\right)$ ). For both the Godunov and Roe fluxes, the locus of intermediate states $\mathbf{u}_{M}$ is the nonphysical branch of the jump conditions through $\mathbf{u}_{R}$ in the interval between $\mathbf{u}_{L}$ and $\mathbf{u}_{R}$. The intermediate states $\mathbf{u}_{M}$ are referred to as equilibrium states. 
Although this family of solutions seems attractive with its minimal number of intermediate states resulting in the sharpest resolved shock, it has the disadvantage of not defining a unique shock position. Using conservation of density or conservation of energy leads to two different estimates of the shock position,

$$
x_{S}(\rho)=\frac{\rho_{M}-\rho_{R}}{\rho_{L}-\rho_{R}} \quad x_{S}(E)=\frac{E_{M}-E_{R}}{E_{L}-E_{R}} .
$$

These estimates will not be the same because, the locus of intermediate states follows a curve. The jump conditions are applied to a given state $\mathbf{u}_{L}$ to find the state $\mathbf{u}_{R}$ behind a stationary shock, and then applied from $\mathbf{u}_{R}$ to a family of states $\mathbf{u}_{M}$. In Figure 1, shock positions from density and energy are plotted against each other, with the deviation from the straight line representing the ambiguity. It turns out that the discrepancy is greater, as might be expected, for stronger shocks, but never exceeds about $5 \%$ of the cell size. While seemingly small, as the jump across the shock itself is $\mathcal{O}(1)$, an $\mathcal{O}(\Delta x)$ error in its position results in a much larger error than a classical error to a cell average in a smooth region of the solution, where the errors are $\mathcal{O}\left((\Delta x)^{2}\right)$.

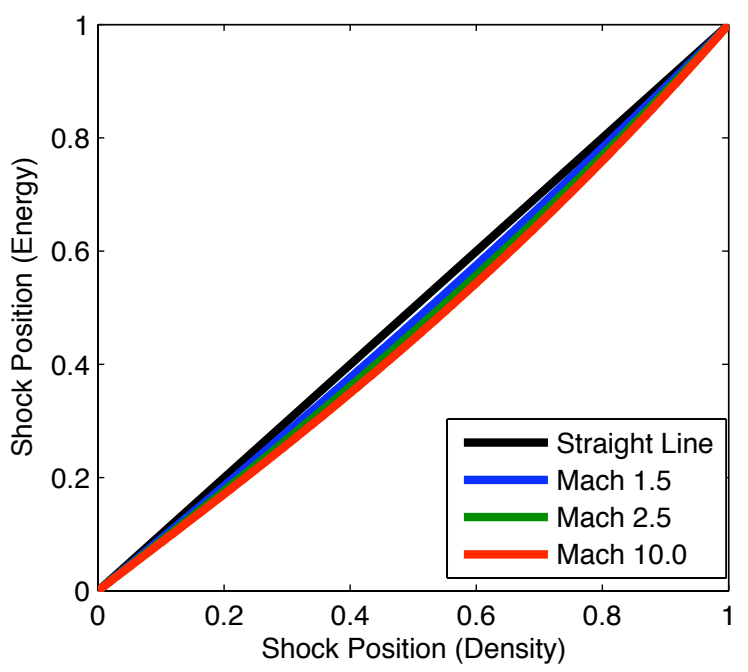

Figure 1: Shock position in energy vs shock position in density in the intermediate cell for initial Mach numbers of $1.5,2.5,4.0$ and 10.0 .

The situation is even worse if shock position is computed using momentum, because as the mass flux, it is equal for the left and right states, but not necessarily for the intermediate state. For the exact Riemann solver or Roe's Riemann solver, a momentum spike of as much as $40 \%$ is possible. In general a spike is observed for all flux functions, independent of the number of intermediate states.

Note that if the jump conditions were linear, there would be no ambiguity present. This ambiguity and nonlinearity has previously been related to each anomaly examined. ${ }^{3}$ It has been noted by Arora and Roe ${ }^{5}$ that the slowly moving shockwave phenomenon was not present for systems with linear jump conditions $\left(\right.$ Temple systems ${ }^{12}$ ). Our goal therefore was to make the Euler equations look like a Temple system. We aimed to devise a scheme admitting a family of steady shocks with an unambiguous sub-cell shock position, consisting of a single intermediate state given by

$$
\mathbf{u}_{M}=\alpha \mathbf{u}_{L}+(1-\alpha) \mathbf{u}_{R}
$$

Such a solution would, incidentally, be the projection of the exact solution into the finite-volume space of piecewise constant functions. We believe that the above objective cannot be satisfied by any Riemann solver $\mathbf{f}_{i+\frac{1}{2}}=\mathbf{f}\left(\mathbf{u}_{i}, \mathbf{u}_{i+1}\right)$, and that more the general idea of a flux function, $\mathbf{f}_{i+\frac{1}{2}}=\mathbf{f}\left(\mathbf{u}_{i-m}, \ldots, \mathbf{u}_{i+n}\right)$ is needed.

\section{New Flux Functions}

If conventional finite volume schemes produce solutions that contain discrepancies, it maybe asked what aspects may be trustworthy. Within an intermediate state, it seems that the conserved quantities should 
be trusted, if for no other reason than to preserve conservation of the system. However, we should perhaps not trust the flux values, since these assume that a pressure can be calculated from an equation of state, and this in turn implies assuming thermodynamic equilibrium. That assumption may not be tenable since it does not hold within a real physical shock.

\section{IV.A. Interpolated Fluxes}

Our idea was to find the fluxes in the untrustworthy intermediate cells by extrapolation from trustworthy neighbors. These interpolated fluxes are denoted by $\mathbf{f}^{*}$ and are constructed to have the following three properties:

1. If the problem is linear such that the Jacobian matrix $\mathbf{A}(\mathbf{u})$ is constant, then $\mathbf{f}_{i}^{*}=\mathbf{f}_{i}$, the interpolated flux equals the equilibrium flux, $\mathbf{f}\left(\mathbf{u}_{i}\right)$, a desirable property as shockwave anomalies do not arise in linear systems.

2. If the problem is nonlinear, but the data is smooth, then $\mathbf{f}_{i}^{*}=\mathbf{f}_{i}+\mathcal{O}\left((\Delta x)^{2}\right)$, a second-order accurate approximation of the flux in smooth regions.

3. If the problem is nonlinear and has a one-point stationary shock, then $\mathbf{f}_{i}^{*}$ is constant, not only on each side of the shock, but also in the intermediate cell, unlike the equilibrium flux. This reflects constancy of the flux in the exact solution, especially in momentum.

To begin, suppose the flux is extrapolated from one side as

$$
\mathbf{f}_{i}^{*}=\mathbf{f}_{i-1}+\tilde{\mathbf{A}}_{i}\left(\mathbf{u}_{i}-\mathbf{u}_{i-1}\right)
$$

for some choice of flux Jacobian, $\tilde{\mathbf{A}}_{i}$, and extrapolated from the other side as

$$
\mathbf{f}_{i}^{*}=\mathbf{f}_{i+1}-\tilde{\mathbf{A}}_{i}\left(\mathbf{u}_{i+1}-\mathbf{u}_{i}\right) .
$$

These two equations are consistent if

$$
\mathbf{f}_{i+1}-\mathbf{f}_{i-1}=\tilde{\mathbf{A}}_{i}\left(\mathbf{u}_{i+1}-\mathbf{u}_{i-1}\right) .
$$

The simplest matrix having this property is a cell-centered Roe matrix, but spanning three states rather than traditional two. Averaging Equations (6) and (7) leads to a centered construction of the interpolated flux

$$
\mathbf{f}_{i}^{*}=\frac{1}{2}\left(\mathbf{f}_{i-1}+\mathbf{f}_{i+1}\right)-\frac{1}{2} \tilde{\mathbf{A}}_{i-1, i+1}\left(\mathbf{u}_{i+1}-2 \mathbf{u}_{i}+\mathbf{u}_{i-1}\right)
$$

With the interpolated flux defined, it is simple to show that for a captured stationary shock having one intermediate state with no positional ambiguity (Equation (5)), the interpolated flux is identical everywhere. The effect of the interpolation is seen by taking a Taylor series expansion of the centered form in Equation $(9)$;

$$
\mathbf{f}_{i}^{*}=\mathbf{f}_{i}+\frac{(\Delta x)^{2}}{2} \mathbf{A}_{x} \mathbf{u}_{x}+\mathcal{O}\left((\Delta x)^{4}\right)=\mathbf{f}_{i}+\frac{1}{2}(\Delta \mathbf{A})(\Delta \mathbf{u})+\mathcal{O}\left((\Delta x)^{4}\right),
$$

which is a modification to the flux proportional to the product of the derivatives of the flux Jacobian and conserved quantities. The modification vanishes if $\mathbf{A}$ is constant, is second-order in smooth regions, but reduces to order unity near nonlinear shocks, countering the effect in Figure 1.

\section{IV.B. New Flux Function - A}

With interpolated fluxes introduced, we defined a new flux function, described similar to the original Roe framework, referred to as new flux function A,

$$
\mathbf{f}_{i+\frac{1}{2}}^{A}=\frac{1}{2}\left(\mathbf{f}_{i}^{*}+\mathbf{f}_{i+1}^{*}\right)-\frac{1}{2} \operatorname{sign}\left(\tilde{\mathbf{A}}_{i+\frac{1}{2}}\right)\left(\mathbf{f}_{i+1}^{*}-\mathbf{f}_{i}^{*}\right),
$$

which reduces to Roe's Riemann solver if $\mathbf{f}_{i}^{*}=\mathbf{f}_{i}$. This ensures that the new method recovers Roe's method for linear problems.

There is one obvious issue with this formulation. In the diffusion component of the Riemann solver, $\operatorname{sign}\left(\tilde{\mathbf{A}}_{i+\frac{1}{2}}\right)\left(\mathbf{f}_{i+1}^{*}-\mathbf{f}_{i}^{*}\right)$. In this term, since the interpolated flux difference is computed with different quantities than the flux Jacobian and the sign function is discontinuous, the flux function is not continuous either. This is potentially a problem, since small changes to the conserved variables could lead to large changes in the flux, which at this point, does not feel right. 


\section{IV.C. New Flux Function - B}

To overcome the discontinuous nature of flux function A, another flux function, B, is written as

$$
\mathbf{f}_{i+\frac{1}{2}}^{B}=\frac{1}{2}\left(\mathbf{f}_{i}^{*}+\mathbf{f}_{i+1}^{*}\right)-\frac{1}{2}\left|\overline{\mathbf{A}}_{i+\frac{1}{2}}\right|\left(\mathbf{u}_{i+1}-\mathbf{u}_{i}\right)
$$

where $\overline{\mathbf{A}}_{i+\frac{1}{2}}$ is the Roe matrix computed from cells $i-1$ and $i+2$,

$$
\overline{\mathbf{A}}_{i+\frac{1}{2}}\left(\mathbf{u}_{i+2}-\mathbf{u}_{i-1}\right)=\mathbf{f}_{i+2}-\mathbf{f}_{i-1} .
$$

An intuitive interpretation is that the matrix $\overline{\mathbf{A}}$ "explains" the difference of states $\mathbf{u}_{i+1}-\mathbf{u}_{i}$ from the viewpoint of a "bigger picture". Similar to flux function A, this reduces to Roe's Riemann solver for a single jump in data. Physically speaking, both new functions trust the values of the conserved quantities, but do not necessarily trust the equilibrium flux functions, and evaluate them differently in the vicinity of a shock.

\section{Second-Order Framework}

For these methods to become practical, they should be easily integrable into more accurate schemes. Although around strong shocks, higher-order methods reduce to first-order, ${ }^{13}$ the new fluxes should still fit within their framework and perform accurately in smooth regions. While there are many more complex methods for these extensions than the ones shown here, they serve as a first step towards integrating both new flux functions into existing methodologies.

To extend the new flux functions to second-order, a finite volume MUSCL-type reconstruction ${ }^{14}$ is used. This is a logical first attempt, since it requires information from four cells and we can use the same in both new flux functions. In the one-dimensional case, a linear reconstruction of the conserved variables in each cell can be defined as

$$
\mathbf{u}_{i}(x)=\mathbf{u}_{i}+\mathbf{u}_{x, i}\left(x-x_{i}\right)
$$

by introducing functions, $\mathbf{u}_{x, i}$, of the left and right gradients,

$$
\mathbf{u}_{x, i}=\mathbf{u}_{x, i}\left(\frac{\mathbf{u}_{i}-\mathbf{u}_{i-1}}{x_{i}-x_{i-1}}, \frac{\mathbf{u}_{i+1}-\mathbf{u}_{i}}{x_{i+1}-x_{i}}\right) .
$$

While reconstructions of any set of variables (primitive, characteristic, etc) could be used, we only use conserved variables to verify the method.

For the four point flux functions, the interface fluxes can be computed using reconstructed data at the edges, with

$$
\mathbf{f}_{i+\frac{1}{2}}=\mathbf{f}\left(\mathbf{u}_{i-1}, \mathbf{u}_{i}^{+}, \mathbf{u}_{i+1}^{-}, \mathbf{u}_{i+2}\right) .
$$

This is equivalent to using interpolated fluxes defined as $\mathbf{f}_{i}^{*,-}, \mathbf{f}_{i}^{*,+}$ which are "edge" values of the interpolated flux.

$$
\begin{aligned}
& \mathbf{f}_{i}^{*,+}=\frac{1}{2}\left(\mathbf{f}\left(\mathbf{u}_{i-1}\right)+\mathbf{f}\left(\mathbf{u}_{i+1}^{-}\right)\right)-\frac{1}{2} \tilde{\mathbf{A}}\left(\mathbf{u}_{i-1}, \mathbf{u}_{i+1}^{-}\right)\left(\mathbf{u}_{i+1}^{-}-2 \mathbf{u}_{i}^{+}+\mathbf{u}_{i-1}\right) . \\
& \mathbf{f}_{i}^{*,-}=\frac{1}{2}\left(\mathbf{f}\left(\mathbf{u}_{i-1}^{+}\right)+\mathbf{f}\left(\mathbf{u}_{i+1}\right)\right)-\frac{1}{2} \tilde{\mathbf{A}}\left(\mathbf{u}_{i-1}^{+}, \mathbf{u}_{i+1}\right)\left(\mathbf{u}_{i+1}-2 \mathbf{u}_{i}^{-}+\mathbf{u}_{i-1}^{+}\right) .
\end{aligned}
$$

with flux function A written as

$$
\mathbf{f}_{i+\frac{1}{2}}^{A}=\frac{1}{2}\left(\mathbf{f}_{i}^{*,+}+\mathbf{f}_{i+1}^{*,-}\right)-\frac{1}{2} \operatorname{sign}\left(\tilde{\mathbf{A}}\left(\mathbf{u}_{i}^{+}, \mathbf{u}_{i+1}^{-}\right)\right)\left(\mathbf{f}_{i+1}^{*,-}-\mathbf{f}_{i}^{*,+}\right)
$$

and flux function B written similarly, with

$$
\mathbf{f}_{i+\frac{1}{2}}^{B}=\frac{1}{2}\left(\mathbf{f}_{i}^{*,+}+\mathbf{f}_{i+1}^{*,-}\right)-\frac{1}{2}\left|\tilde{\mathbf{A}}\left(\mathbf{u}_{i-1}, \mathbf{u}_{i+2}\right)\right|\left(\mathbf{u}_{i+1}^{-}-\mathbf{u}_{i}^{+}\right) .
$$

This leads to the idea that the interpolated flux, as an independent variable in itself, has two slopes, depending on which edge value is needed. The accuracy of these reconstructions can be analytically verified by noting 
that for smooth data, $\mathbf{f}^{*,+}=\mathbf{f}^{*}\left(\mathbf{u}_{i-1}, \mathbf{u}_{i}^{+}, \mathbf{u}_{i+1}^{-}\right) \approx \mathbf{f}\left(\mathbf{u}_{i}^{+}\right)$, the classical result for a second-order finite volume scheme.

Finally, with the reconstruction defined, to prevent oscillations produced by the reconstruction, limiters are required. A well known problem with second-order and higher schemes, ${ }^{15}$ these oscillations are quite different than those produced in the first-order scheme, but nonetheless problematic. Limiting within the new flux functions is done identically to that of more traditional Riemann solvers, by applying limiters to the slopes of the reconstructions of the conserved variables.

\section{Numerical Results}

Numerical results are shown for a series of smooth problems as well as on problems with numerical shockwave anomalies. This will verify the second-order performance while ensuring the second-order method still reduces anomalous behavior in the vicinity of shocks. To prevent temporal errors from reducing spatial accuracy, the following second-order Runge-Kutta method is used ${ }^{16}$

$$
\begin{aligned}
\mathbf{u}_{i}^{n+\frac{1}{2}} & =\mathbf{u}_{i}^{n}+\frac{\Delta t}{\Delta x}\left(\mathbf{f}_{i+\frac{1}{2}}^{n}-\mathbf{f}_{i-\frac{1}{2}}^{n}\right) \\
\mathbf{u}_{i}^{n+1} & =\frac{1}{2}\left(\mathbf{u}_{i}^{n}+\mathbf{u}_{i}^{n+\frac{1}{2}}\right)+\frac{\Delta t}{2 \Delta x}\left(\mathbf{f}_{i+\frac{1}{2}}^{n+\frac{1}{2}}-\mathbf{f}_{i-\frac{1}{2}}^{n+\frac{1}{2}}\right) .
\end{aligned}
$$

\section{VI.A. Smooth Problems}

To verify the accuracy of the second-order method, several simple wave tests are performed. The first is a simple traveling density wave with solution

$$
\rho=\rho_{\infty}+A \sin \left[2 \pi\left(x-u_{\infty} t\right)\right], \quad u=u_{\infty}, \quad p=p_{\infty}
$$

on a periodic domain of $0 \leq x \leq 1$. In the results shown, $A=0.5$ with reference quantities $\rho_{\infty}=u_{\infty}=$ $p_{\infty}=1$. A CFL number of 0.8 is used and the error is measured at a time of $t=1.0$, corresponding to one complete period in $x$. The spatial error is computed from the exact solution using the L-2 norm of density. The results in Figure 2a demonstrate that second-order accuracy is achieved for both new flux functions, which are indistinguishable in the convergence plot and behave identically to that of Roe's Riemann solver.

Next, two simple acoustic waves described by Lowrie ${ }^{17}$ with initial conditions

$$
\begin{aligned}
\frac{u(x)}{a_{\infty}} & =M_{\infty}+\frac{2}{\gamma+1} \frac{V(x)}{a_{\infty}} \\
\frac{\rho(x)}{\rho_{\infty}} & =\left[1+\frac{\gamma-1}{2}\left(\frac{u(x)}{a_{\infty}}-M_{\infty}\right)\right]^{\frac{2}{\gamma-1}} \\
\frac{p(x)}{p_{\infty}} & =\left[1+\frac{\gamma-1}{2}\left(\frac{u(x)}{a_{\infty}}-M_{\infty}\right)\right]^{\frac{2 \gamma}{\gamma-1}}
\end{aligned}
$$

are used, where $V=u+a$, and $V$ is a solution to Burgers' equation. First, a smooth expansion wave is examined, with initial condition

$$
V(x)=0.04 \tanh (10(x-0.5)) \text {. }
$$

The reference Mach number, $M_{\infty}$, is set to $-\sqrt{\gamma}$ to keep the expansion wave centered inside the domain. A CFL number of 0.5 is used and a similar set of results to the previous tests are generated. In Figure $2 \mathrm{~b}$, first and second-order results are presented.

As second-order accuracy is achieved for the expansion wave, a smooth solution sharpening into a shockwave, with initial condition

$$
V(x)=\frac{1}{2 \pi t_{s}} \cos (2 \pi x)
$$

is examined, where $t_{s}$ corresponds to the time of shock formation. The reference quantities are $M_{\infty}=a_{\infty}=$ $\rho_{\infty}=p_{\infty}=1.0$ and shock formation time is 2.0. A smooth solution is required for accuracy, so the spatial error is measured prior to the shock formation, at $t=1.0$, when the solution is still relatively smooth. Again, a CFL number of 0.8 is used on each domain and the L-2 norm of density error is measured. Results in Figure 2c demonstrate second-order accuracy similar to that of the other two simple waves. 


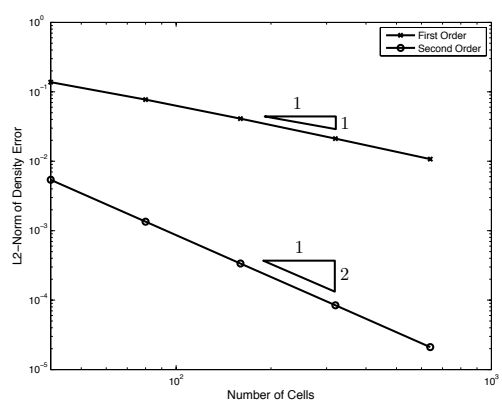

(a) Density wave.

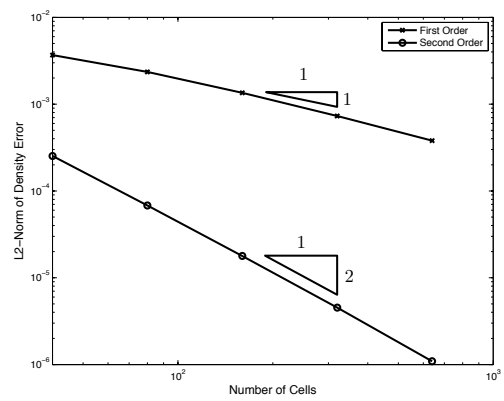

(b) Expansion wave.

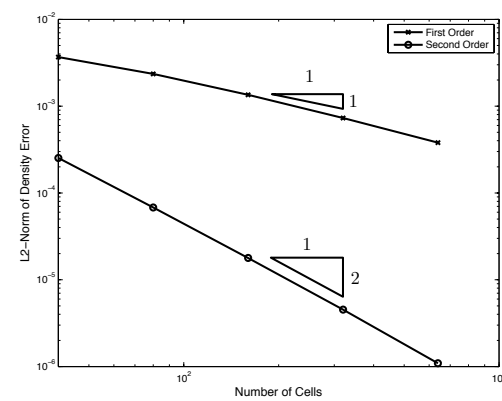

(c) Compression wave.

Figure 2: L2-Norm of Density Error Convergence for 1D smooth problems. Second-order accuracy is obtained using the new flux functions, which behave nearly identically in each problem, an expected result for smooth data.

\section{VI.B. Slowly Moving Shockwave}

The slowly moving shockwave phenomenon is one of the aforementioned numerical methods and is characterized by the shedding of spurious waves by slowly moving shocks. These waves are purely numerical, but once created, they are propagated as though they were real. The cause of this phenomenon is that not all waves produced by solving Riemann problems involving the intermediate states are of the same family as the shockwave. While the term 'slowly moving' can be interpreted in many ways, it is generally used to refer to shocks where the characteristics change sign across the shock, although in practice this is not a necessary condition.

For the numerical results here, the slowly moving shockwave is initialized as a shocktube problem with left and right states given by

$$
\left[\begin{array}{c}
\rho_{L} \\
u_{L} \\
p_{L}
\end{array}\right]=\left[\begin{array}{c}
1 \\
1 \\
\frac{1}{\gamma M_{L}^{2}}
\end{array}\right], \quad\left[\begin{array}{c}
\rho_{R} \\
u_{R} \\
p_{R}
\end{array}\right]=\left[\begin{array}{c}
\frac{S-1}{S-\frac{2(S-1)}{\gamma+1}+\frac{2}{M_{L}^{2}(\gamma+1)(S-1)}-1} \\
\frac{2(S-1)}{\gamma+1}-\frac{2}{M_{L}^{2}(\gamma+1)(S-1)}+1 \\
\frac{2(S-1)^{2}}{\gamma+1}-\left(\frac{2}{\gamma+1}-\frac{1}{\gamma}\right) \frac{1}{M_{L}^{2}}
\end{array}\right] .
$$

To compare the effectiveness of the new flux functions over a range of shock speeds, the grid normalized shock speed, $S \Delta t / \Delta x$, is varied and the size of the momentum spike is measured as

$$
\text { Relative Maximum Momentum Error }=\frac{\max _{x, t}(\rho u)-\rho_{R} u_{R}}{\rho_{R} u_{R}} .
$$

Momentum error plots for Mach 2 and Mach 10 shockwaves are shown in Figure 5 with three well known limiter functions: double minmod, ${ }^{18}$ minmod, ${ }^{19}$ and the harmonic limiter (van Leer). ${ }^{20}$ For slow shock speeds, both flux functions A and B dramatically reduce the error when compared to Roe's Riemann solver, in both the first and second-order schemes. This improvement is greatest at higher Mach numbers, where flux function A displays practically no momentum spike at all. Examining individual cases in Figures 3 reveals that the error is reduced without producing additional intermediate states, resulting in a sharp shockwave with minimal error. As usual, the second-order schemes performed worse than the first-order one, as they have less dissipation and resolve the spurious waves (and momentum spike) more accurately.

\section{VI.C. One Dimensional Carbuncle}

Both methods A and B are designed to capture stationary shocks with one intermediate point described by (5) and indeed they do. It does need to be asked, however, whether those solutions are stable. It was established by Barth, and by Serre, ${ }^{7,21}$ that the one-point shocks produced by the Godunov and Roe methods are not always stable. The instability happens for strong enough shocks, over a range of shock locations. Depending on the boundary conditions, the shock will either move to a stable position or enter an 


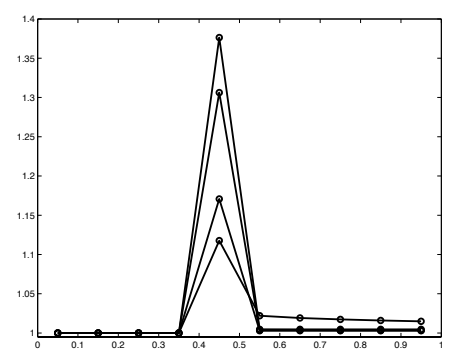

(a) Roe, $1^{\text {st }}$-order

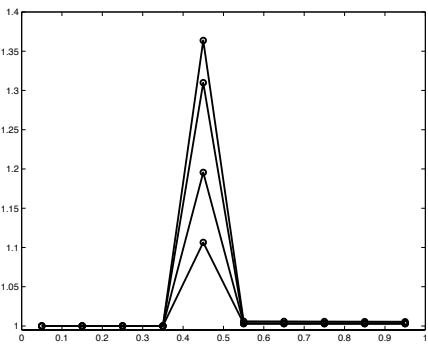

(d) Roe, $2^{\text {nd }}$-order

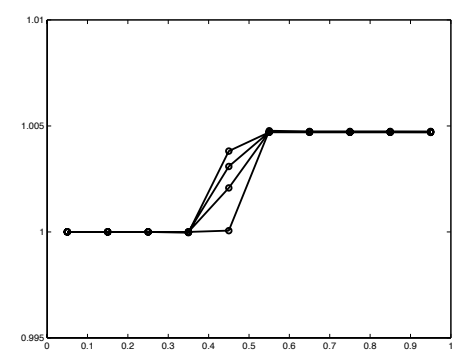

(b) Flux A, $1^{\text {st }}$-order

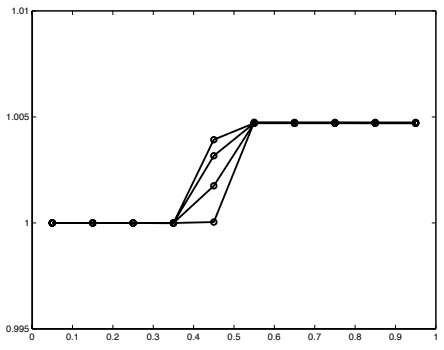

(e) Flux A, $2^{\text {nd }}$-order

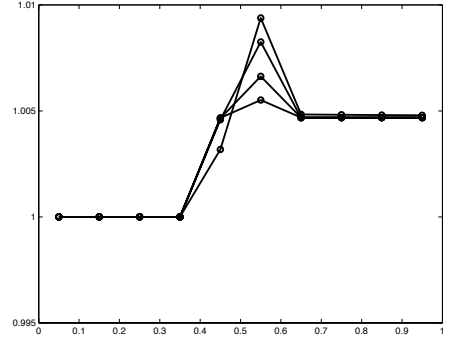

(c) Flux B, $1^{\text {st }}$-order

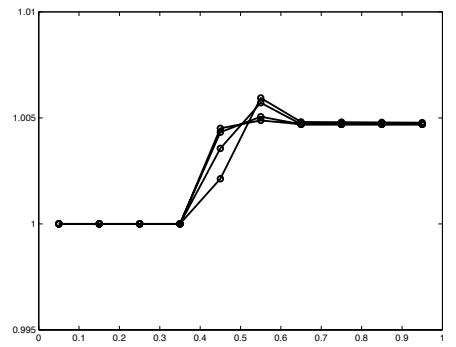

(f) Flux B, $2^{\text {nd }}$-order

Figure 3: Snapshots of momentum at four equally spaced times for a slowly moving shock of Mach 10 and $S \Delta t / \Delta x=0.002$, corresponding to $\rho_{L} u_{L}=1, \rho_{R} u_{R}=1.005$. Representative results are shown for the first-order method and second-order method with minmod limiter. The momentum spike for Roe's Riemann solver is $\sim 40 \%$, while both new fluxes have no or minimal momentum spike.. For each flux function, the shock is sharply resolved with one or two intermediate states.

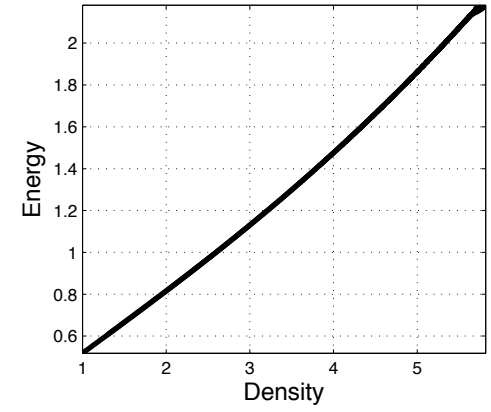

(a) Roe, $1^{\text {st }}$-order

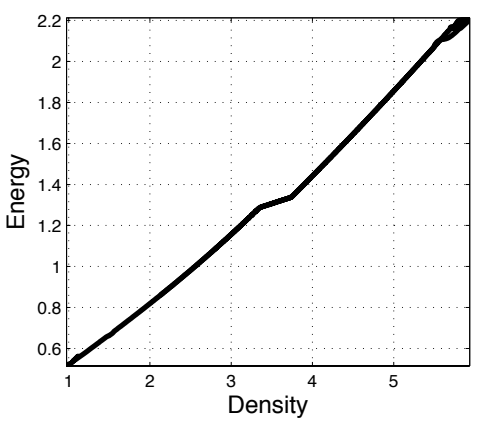

(d) Roe, $2^{\text {nd }}$-order

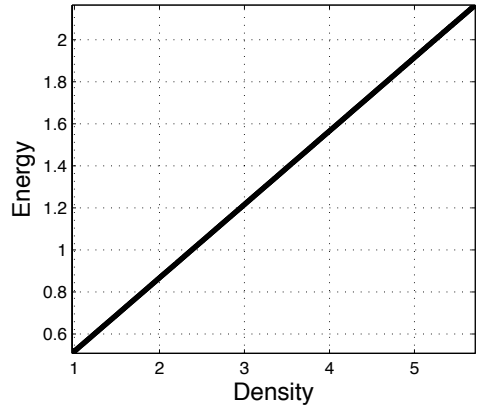

(b) Flux A, $1^{\text {st }}$-order

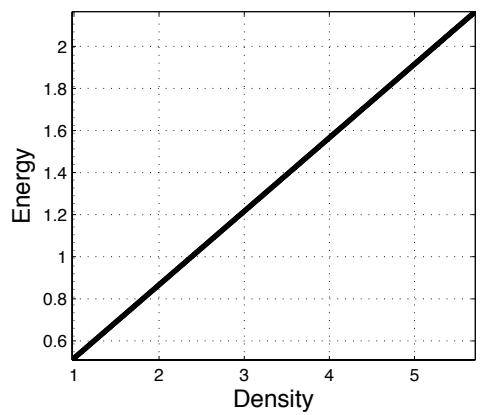

(e) Flux A, $2^{\text {nd }}$-order

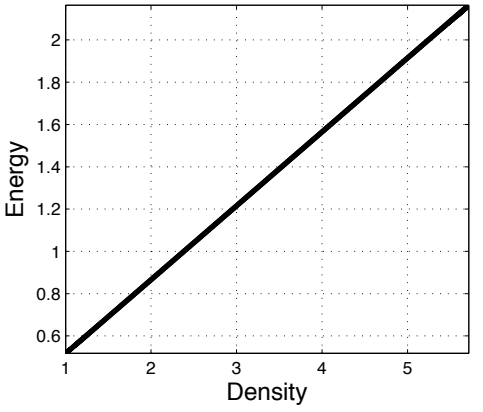

(c) Flux B, $1^{\text {st }}$-order

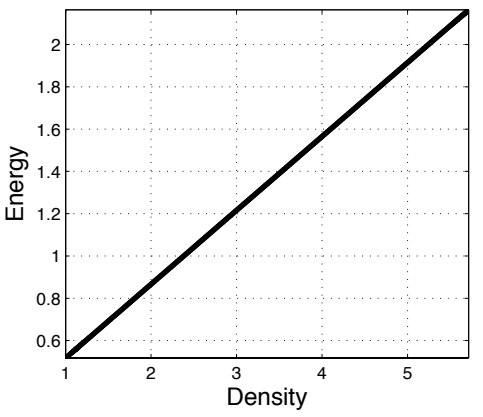

(f) Flux B, $2^{\text {nd }}$-order

Figure 4: Density-Energy plots for the slowly moving shock problem described in Figure 3. Representative results are shown for the first-order method and second-order method with minmod limiter. Both new flux functions are much closer to the straight line than Roe's Riemann solver. 


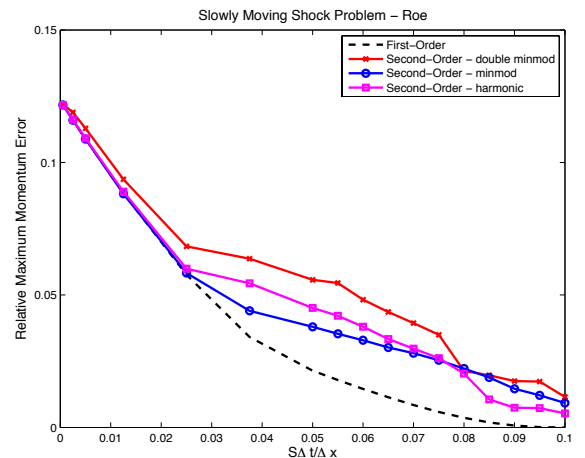

(a) Roe, Mach 2

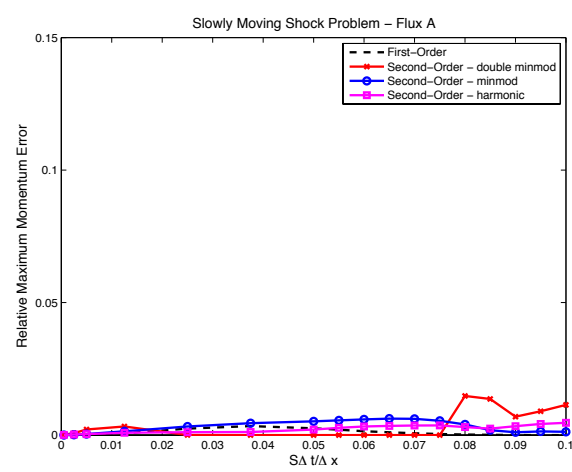

(c) Flux A, Mach 2

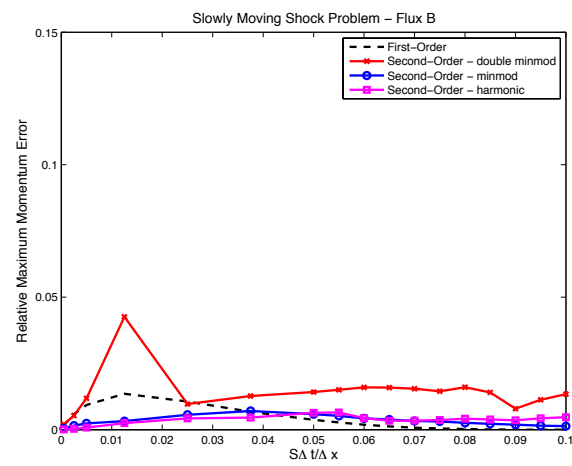

(e) Flux B, Mach 2

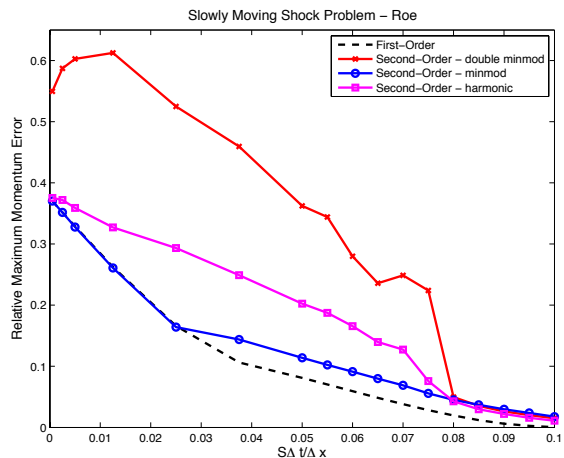

(b) Roe, Mach 10

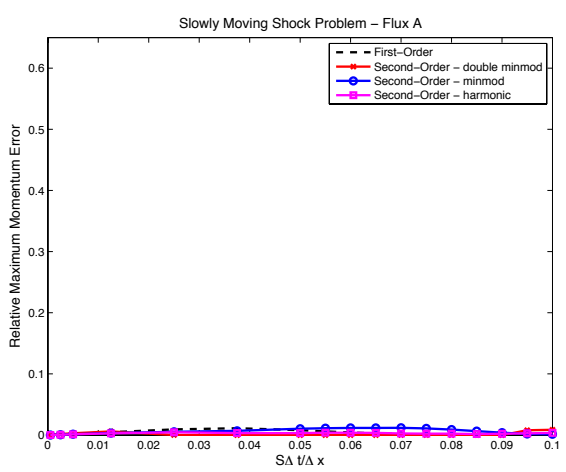

(d) Flux A, Mach 10

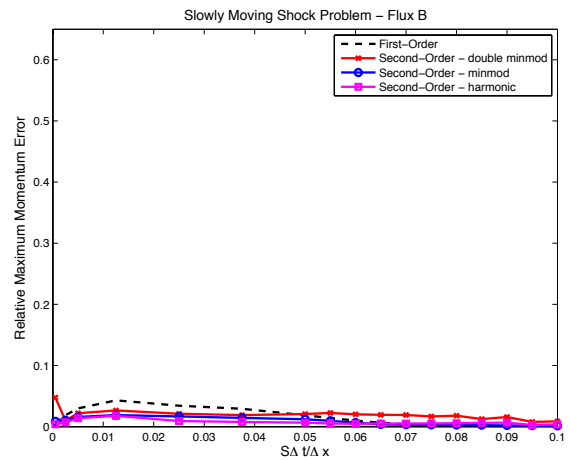

(f) Flux B, Mach 10

Figure 5: The relative momentum error plotted for a range of grid normalized shock speeds $(S \Delta t / \Delta x)$ for a Mach 2 shock (top) and Mach 10 shock (both) for the first-order method and the second-order method with three well known slope limiters. Results for Flux A are on the left, Flux B in the middle, and Roe's Riemann solver on the right.

approximate limit cycle. This behavior has been called a "one-dimensional carbuncle". No evidence of this is found in experiments on flux functions A or B, and this is confirmed by a numerical eigenvalue analysis, along the lines conducted by Barth.

Starting with a one-point stationary shock with left and right states described in Equation(29) with $S=0$, the residual function corresponding to the net flux through the intermediate state is

$$
\mathbf{r}\left(\mathbf{u}_{M} ; \mathbf{u}_{L}, \mathbf{u}_{R}\right)=\mathbf{f}_{M R}-\mathbf{f}_{L M}
$$

for the shock with left and right endpoints $\mathbf{u}_{L}$ and $\mathbf{u}_{R}$. This function has a zero eigenvalue and a singular Jacobian matrix, $\operatorname{det}\left[\frac{\partial \mathbf{r}}{\partial \mathbf{u}_{M}}\right]=0$, since all middle states $\mathbf{u}_{M}$ are stationary points. To determine the 
sensitivity to the zero eigenvalue, examine the semi-discrete equations on a grid with unit spacing,

$$
\frac{\partial \mathbf{u}_{M}}{\partial t}+\mathbf{r}\left(\mathbf{u}_{M} ; \mathbf{u}_{L}, \mathbf{u}_{R}\right)=\mathbf{0}
$$

Near a stationary solution, $\mathbf{u}^{*}$, the residual, $\mathbf{r}\left(\mathbf{u}^{*} ; \mathbf{u}_{L}, \mathbf{u}_{R}\right)$, is zero and the residual can be linearized with $\mathbf{u}_{M}=\delta \mathbf{u}+\mathbf{u}^{*}$ as

$$
\mathbf{r}\left(\mathbf{u}_{M}\right)=\mathbf{r}\left(\mathbf{u}^{*}+\delta \mathbf{u}\right)=\mathbf{r}\left(\mathbf{u}^{*}\right)+\frac{\partial \mathbf{r}\left(\mathbf{u}^{*}\right)}{\partial \mathbf{u}_{M}} \delta \mathbf{u}
$$

such that $\delta \mathbf{u}$ is governed by

$$
\frac{\partial(\delta \mathbf{u})}{\partial t}+\frac{\partial \mathbf{r}\left(\mathbf{u}^{*}\right)}{\partial \mathbf{u}_{M}} \delta \mathbf{u}=0
$$

and the solution can be determined from the eigenvalues, $\lambda_{i}$, and eigenvectors, $\mathbf{r}_{i}$, of the residual Jacobian,

$$
\delta \mathbf{u}(t)=\alpha_{1} \mathbf{r}_{1}+\alpha_{2} \mathbf{r}_{2} e^{-\lambda_{2} t}+\alpha_{3} \mathbf{r}_{3} e^{-\lambda_{3} t}
$$

where $\lambda_{1}$ corresponds to the stationary eigenvalue and is zero. Stability and carbuncle free requires $\lambda_{2}, \lambda_{3} \geq 0$ to ensure the stability of $\mathbf{u}_{M}$. To avoid boundary effects, the eigenvalues of $\frac{\partial \mathbf{r}}{\partial \mathbf{u}}$ are examined for a stationary shock problem using several extra cells. For this analysis, the initial middle state is created on the nonphysical branch of the jump conditions for Roe's Riemann solver and on a straight line in state space for the new flux functions. To examine stability for the second-order methods, the middle state is reconstructed and the slopes are used in the construction of $\mathbf{f}_{M R}-\mathbf{f}_{L M}$, which become $\mathbf{f}_{\left(M^{+}\right) R}-\mathbf{f}_{L\left(M^{-}\right)}$.

The results are shown in Figure 6 for Roe's flux function and both new flux functions. For the secondorder method, only results for the minmod limiter are shown as other limiters produce nearly identical results. At Mach 10 and above, negative eigenvalues are seen only for the Roe flux. From this analysis, both new flux functions are carbuncle-free in one dimension in both first and second-order methods.

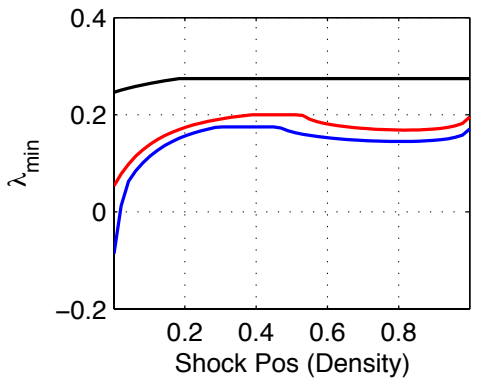

(a) Roe, $1^{\text {st }}$-order

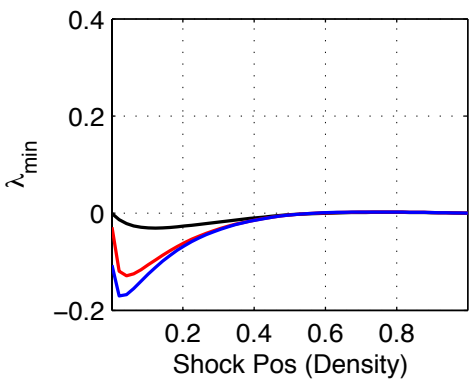

(d) Roe, $2^{\text {nd }}$-order

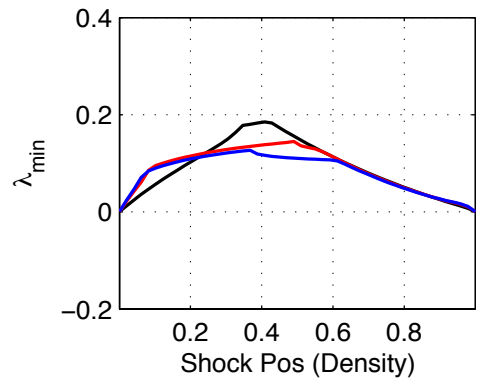

(b) Flux A, $1^{\text {st }}$-order

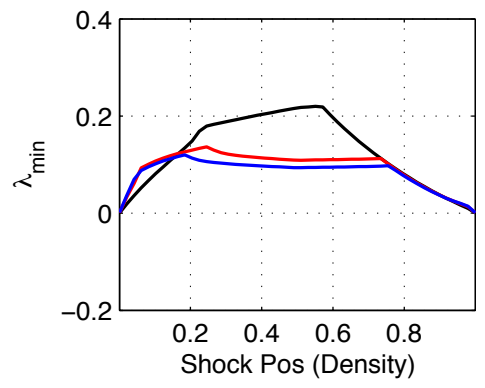

(e) Flux A, $2^{\text {nd }}$-order

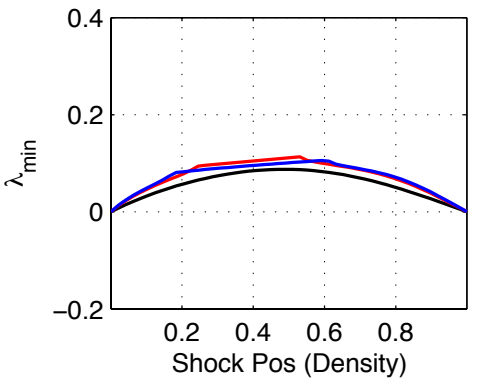

(c) Flux B, $1^{\text {st }}$-order

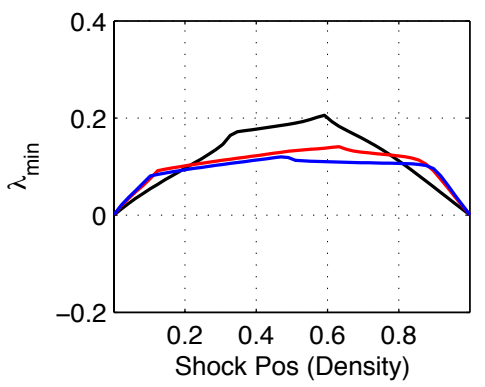

(f) Flux B, $2^{\text {nd }}$-order

Figure 6: Representative stability results for the one-dimensional carbuncle for Mach 2 (black), Mach 5 (red) and Mach 10 (blue) using a first-order method and a second-order method with minmod limiter. Only Roe's Flux has negative (unstable) eigenvalues, in both the first and second-order method.

\section{VI.D. Noh Problem}

With the impressive performance on the slowly moving shock problem and the carbuncle, the new fluxes are tested on the Noh Problem, ${ }^{22}$ where the outcome is less satisfying. The initial data is $\mathbf{u}_{0}=\left(\rho_{0},-\rho_{0} u_{0}, E_{0}\right)^{T}$ 
but can be reduced to a one parameter family with Mach number as the free parameter and

$$
\rho_{0}=1, \quad u_{0}=1, \quad p_{0}=\frac{1}{\gamma M_{0}^{2}} .
$$

The solution behind the shock can be determined exactly from the jump conditions as

$$
\rho=1+\frac{1}{S}, \quad u=0, \quad p=p_{0}+(1+S),
$$

with shock speed

$$
S=\frac{1}{4}\left((\gamma-3)+\sqrt{(\gamma+1)^{2}+16 \gamma p_{0}}\right) .
$$

Results are shown for a CFL number of 0.5. While there is some dependency on CFL number, the trends displayed and overall performance remains the same. Density from the new fluxes is compared to Roe's Riemann solver in Figure 7 (all three methods compute velocity and pressure equivalently and results are not shown). Looking at the density defect at the wall, flux function A dramatically outperforms flux function B, especially for the Mach 10 shock. Two measures of error are used for comparing methods. First, the relative wall density error, defined as

$$
\text { Relative Wall Density Error }=\frac{\rho_{\text {wall }}-\rho_{\text {exact }}}{\rho_{\text {exact }}}
$$

is used as a measure of the defect exactly at the wall. On the left in Figure 8, the results show that flux function A provides a significant improvement to the error at the wall, almost eliminating it with the secondorder method. Flux function B performs comparably to Roe's method, with a slight improvement in the first-order scheme and equal performance in the second-order scheme.
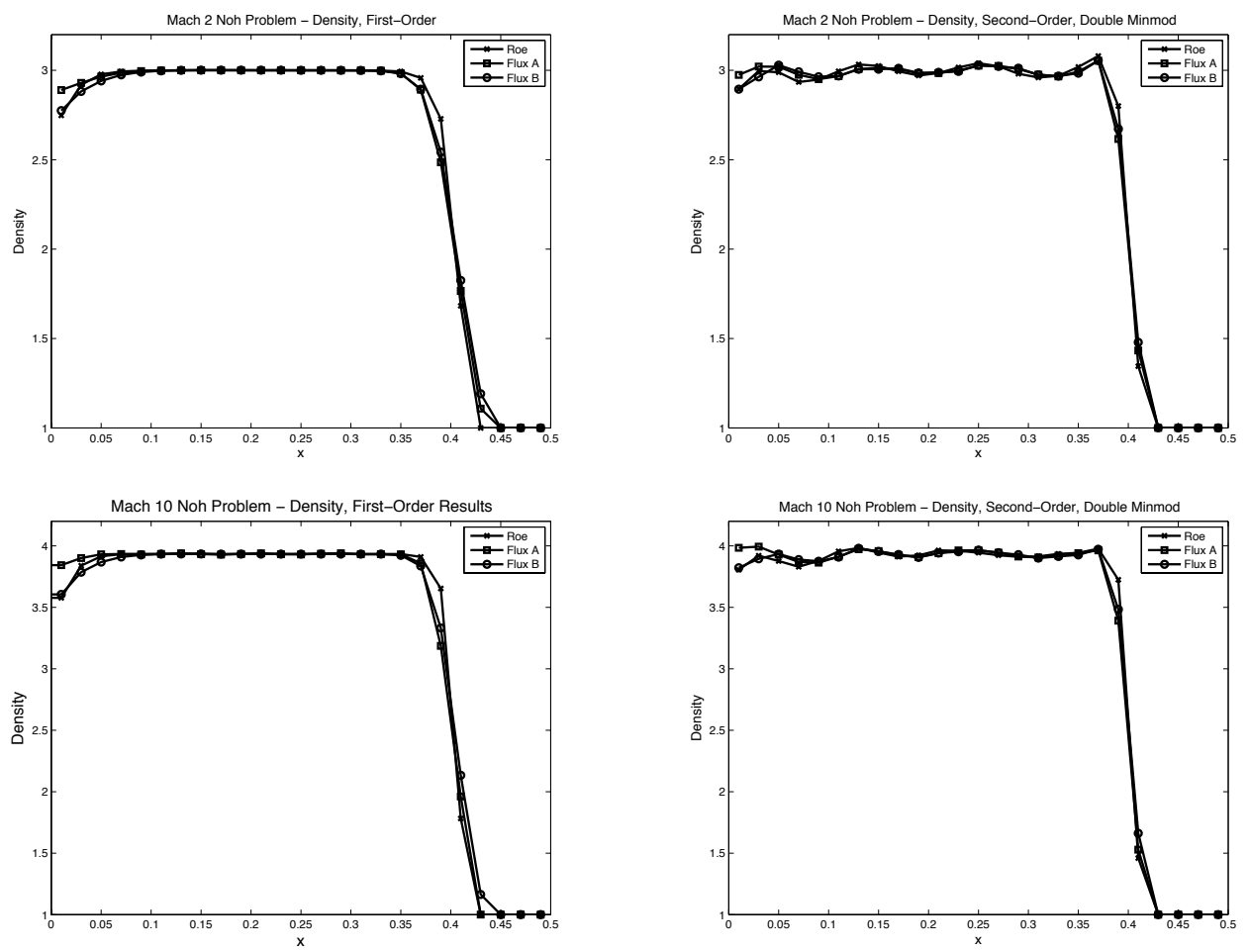

Figure 7: Results for the Noh Problem for Mach 2 (top) and Mach 10 (bottom). Representative results are shown for the first and second-order methods, with the double minmod limiter used. For all flux functions, the second-order scheme preserves the spurious waves much better than the first-order scheme, resulting in oscillatory behavior behind the shock. 
With flux function A performing well, a second estimate of error is used, the total error behind the shock, measured as the L-1 Norm of the relative density error at each point behind the shock,

$$
\text { Total Error Behind the Shock }=\frac{\sum\left|\rho-\rho_{\text {exact }}\right| \Delta x}{\rho_{\text {exact }}} .
$$

This is used to ensure that if our new flux functions reduce the error at the wall, they are not reducing it by compensating with additional errors behind the shock, but rather correcting the internal shock structure. On the right in Figure 8 shows that the first-order flux A is actually reducing the error, while the second-order scheme merely re-distributes the error. By conservation, this suggests the ambiguity in shock position has not been significantly improved by either new flux function. This is not a surprising result given that the shock is far from stationary and contains multiple intermediate states, however, it makes doubtful our earlier claim to have substantially reduced the wall heating error.

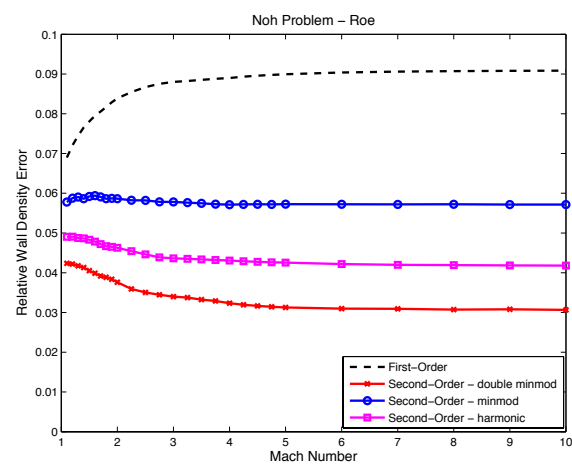

(a) Roe

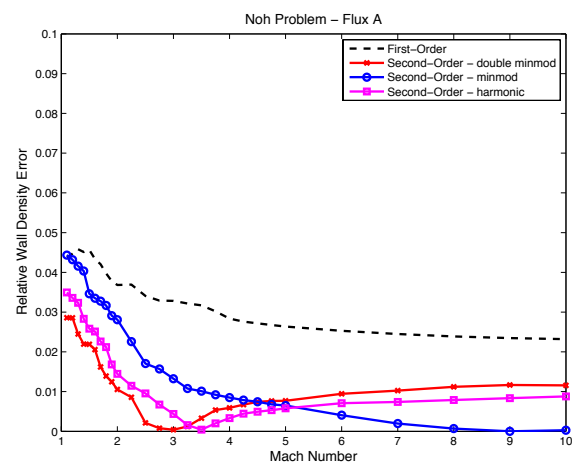

(c) Flux A

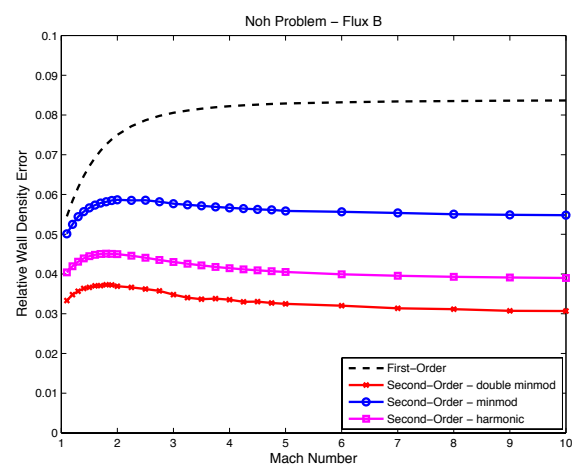

(e) Flux B

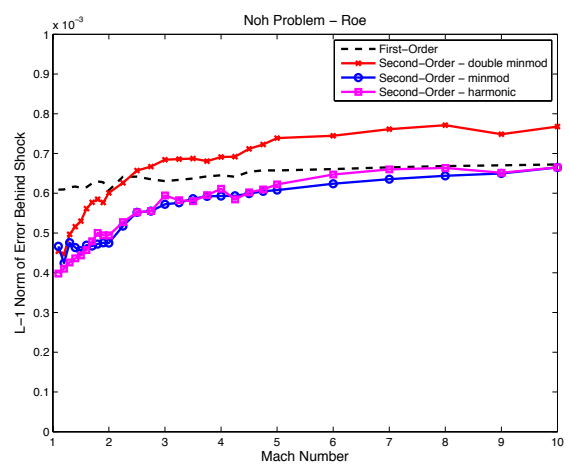

(b) Roe

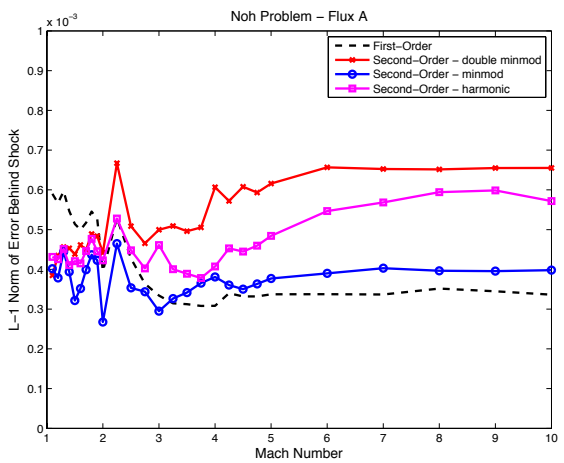

(d) Flux A

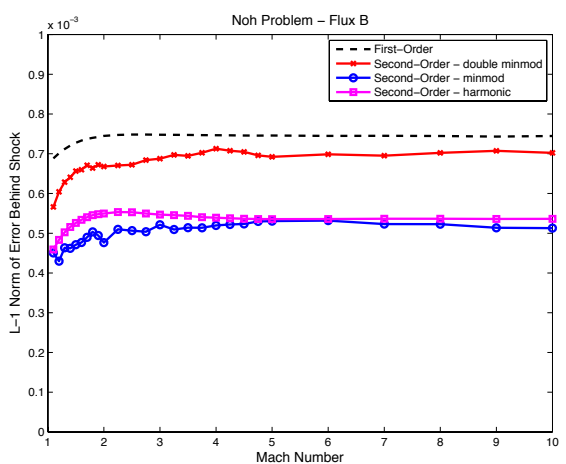

(f) Flux B

Figure 8: The relative wall density error (left) and total error behind the shock (right) for the Noh problem for a range of Mach numbers for both the first and second-order methods. 


\section{VI.E. Shu-Osher Problem}

The final problem examined, the Shu-Osher problem, ${ }^{23}$ is a well known test problem where a Mach 3 shockwave interacts with a wave in density designed to test how well numerical methods resolve small scale flow features in the presence of shocks. While generally used as a test for higher-order methods, it is still a good test for the new flux functions to see how well they handle interacting shocks and contact discontinuities. A domain of $-5 \leq x \leq 5$ is used with left and right states separated at $x=-4$ and initial conditions

$$
\left[\begin{array}{l}
\rho_{L} \\
u_{L} \\
p_{L}
\end{array}\right]=\left[\begin{array}{l}
3.857143 \\
2.629369 \\
10.33333
\end{array}\right], \quad\left[\begin{array}{l}
\rho_{R} \\
u_{R} \\
p_{R}
\end{array}\right]=\left[\begin{array}{c}
1.0+0.2 \sin (5 x) \\
0.0 \\
1.0
\end{array}\right] .
$$

and $\gamma=1.4$. Two hundred cells are used with a CFL of 0.8. Again, both new flux functions perform comparably to Roe's Riemann solver, as shown in Figure 9 for both density and pressure. The results in this problem and the previous two problems are not all that surprising given the new fluxes resemble Roe's Riemann solver outside of stationary or slowly moving shocks.

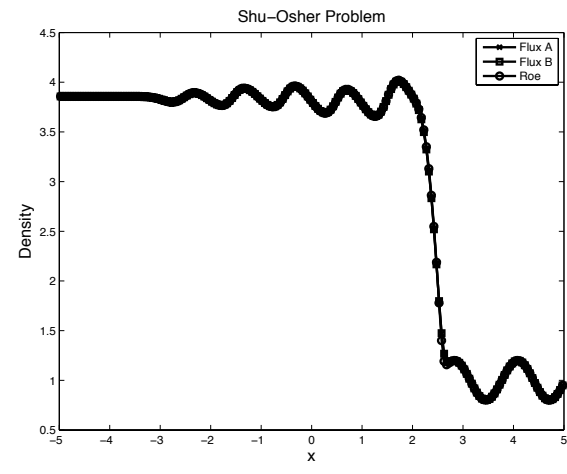

(a) $1^{\text {st }}$ Order, Density

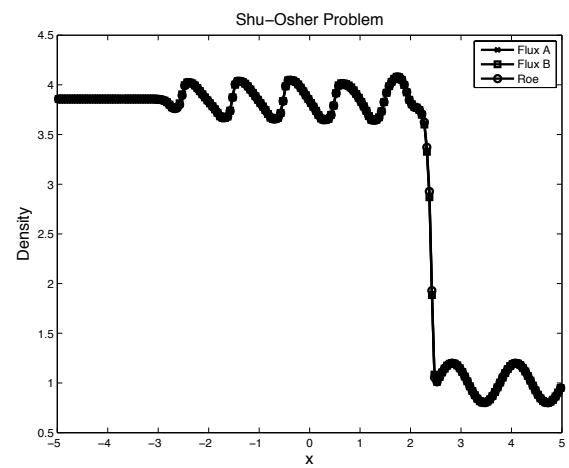

(c) $2^{\text {nd }}$ Order, Density, Harmonic Limiter

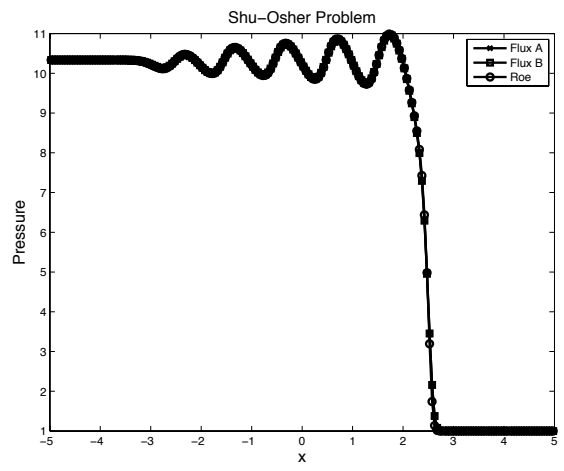

(b) $1^{\text {st }}$ Order, , Pressure

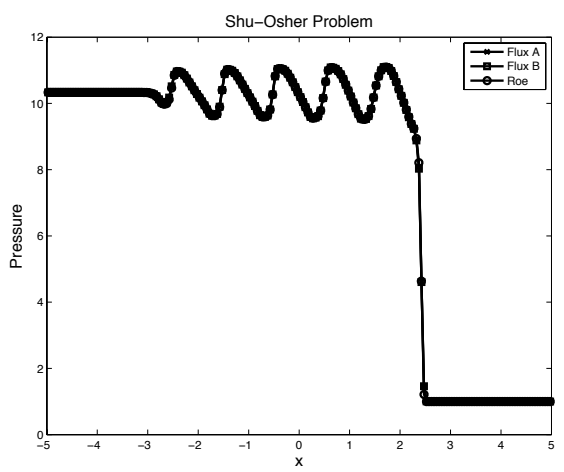

(d) $2^{\text {nd }}$ Order, Pressure, Harmonic Limiter

Figure 9: First and second-order results for the Shu-Osher problem for Flux A, Flux B, and Roe.

\section{Conclusions and Future Work}

In this work, two new flux functions that reduce shockwave anomalies were tested on a range of smooth problems using a MUSCL-type reconstruction to obtain second-order accuracy. This was expected since for smooth problems, the interpolated flux is merely a second-order correction to the exact flux, such that the effect of both new flux functions is minimal. The extension to second-order allows for the existing four states used in both new flux functions to be reconstructed for accuracy while maintaining a reduction in shockwave anomalies, notably the slowly moving shock phenomenon and one dimensional carbuncle. On problems with shockwaves, standard slope limiting techniques were shown to maintain the performance of both new flux functions on numerical shockwave anomalies, providing increased accuracy in smooth regions 
while minimizing oscillations around shockwaves. This work also provides guidance on how to better utilize additional information such as slopes and allows for additional new flux functions to be developed that maintain an unambiguous shock structure.

There are potentially numerous alternative flux formulae, based on the interpolated fluxes, able to preserve the same desirable steady shock structures, and these are actively being investigated, though with mixed results. However, the most urgent task is to extend the work to higher dimensions. A starting point for this is to consider a stationary shockwave oblique to the mesh. We can declare that no cell intersected by this shock is trustworthy, and try to replace the flux in such cells by interpolations, all of which should lie on the straight line in state space that joins the preshock and postshock states.

A two dimensional implementation was included in the dissertation Numerical Shockwave Anomalies. ${ }^{10}$ On a structured grid, an interpolated flux was computed in each direction within a second-order finite volume method, a natural extension of the one dimensional version. Unfortunately, the method tended to be unstable in regions where velocities changed sign, amplifying small oscillations and often resulting in non-physical solutions near stagnation or recirculation regions as well as tangential to shockwaves, when the tangential velocity is small. These phenomena seem unrelated to the thermodynamic issues investigated here, leading us to suspect yet another ingredient is needed for successful shock capturing in hypersonic flow. When the method behaved, both new flux functions substantially reduced the spurious waves produced by the two dimensional slowly moving shock phenomenon, however, with the lack of robustness, we do not demonstrate results at this time. These positive results are encouraging though, and we are actively working on methods to overcome the current instabilities and dramatically reduce two dimensional shockwave anomalies.

\section{Acknowledgments}

The first author would like to thank Professor Carl F. Ollivier-Gooch at the University of British Columbia for financial support.

\section{References}

${ }^{1}$ Salas, M. D., "A Shock-Fitting Primer," 2010, pp. 1-397.

${ }^{2}$ Menikoff, R., "Errors when shock waves interact due to numerical shock width," SIAM Journal on Scientific Computing, Vol. 15, No. 5, 1994, pp. 1227-1242.

${ }^{3}$ Zaide, D. W. and Roe, P. L., "Shock Capturing Anomalies and the Jump Conditions in One Dimension," Proceedings of 20th AIAA Computational Fluid Dynamics Conference, 2011-3686, 2011.

${ }^{4}$ Roberts, T. W., "The behavior of flux difference splitting schemes near slowly moving shock waves," Journal of Computational Physics, Vol. 90, No. 1, 1990, pp. 141-160.

${ }^{5}$ Arora, M. and Roe, P. L., "On postshock oscillations due to shock capturing schemes in unsteady flows," Journal of Computational Physics, Vol. 130, No. 1, 1997, pp. 25-40.

${ }^{6}$ Karni, S. and Canic, S., "Computations of slowly moving shocks," Journal of Computational Physics, Vol. 136, No. 1, 1997, pp. 132-139.

${ }^{7}$ Barth, T. J., "Some notes on shock resolving flux functions. Part 1: Stationary characteristics," NASA Technical Memorandum, 1989.

${ }^{8}$ Pandolfi, M. and D'Ambrosio, D., "Numerical instabilities in upwind methods: analysis and cures for the "Carbuncle" Phenomenon," Journal of Computational Physics, Vol. 166, No. 2, 2001, pp. 271-301.

${ }^{9}$ Bressan, A., Jenssen, H., and Baiti, P., "An instability of the Godunov scheme," Communications on Pure and Applied Mathematics, Vol. 59, No. 11, 2006, pp. 1604-1638.

${ }^{10}$ Zaide, D. W., "Numerical Shockwave Anomalies," Ph.D. Thesis, May 2012, pp. 1-112.

${ }^{11}$ Toro, E. F., "Riemann Solvers and Numerical Methods for Fluid Dynamics: A Practical Introduction," Springer-Verlag, 1999, pp. 1-605.

${ }^{12}$ Temple, B., "Systems of conservation laws with invariant submanifolds," Transactions of the Americal Mathematical Society, Vol. 280, No. 2, 1983, pp. 781-795.

${ }^{13}$ Carpenter, M. H. and Casper, J. H., "Accuracy of shock capturing in two spatial dimensions," AIAA Journal, 1999, pp. $488-498$.

${ }^{14}$ van Leer, B., "Towards the ultimate conservative difference scheme. IV. A new approach to numerical convection," Journal of Computational Physics, Vol. 23, 1977, pp. 276-299.

${ }^{15}$ Godunov, S. K., "A difference method for numerical calculation of discontinuous solutions of the equations of hydrodynamics," Matematicheskii Sbornik, 1959.

${ }^{16}$ Gottlieb, S., Shu, C.-W., and Tadmor, E., "Strong stability-preserving high-order time discretization methods," SIAM review, Vol. 43, No. 1, 2001, pp. 89-112.

${ }^{17}$ Lowrie, R. B., "Compact Higher-Order Numerical Methods for Hyperbolic Conservation Laws," Ph.D. Thesis, 1996. 
${ }^{18}$ van Leer, B., "Towards the ultimate conservative difference scheme. V. A second-order sequel to Godunov's method," Journal of Computational Physics, Vol. 32, No. 1, 1979, pp. 101-136.

${ }^{19}$ Roe, P. L., "Characteristic-based schemes for the Euler equations," Annual review of fluid mechanics, Vol. 18, Dec 1986, pp. 337-365.

${ }^{20}$ van Leer, B., "Towards the ultimate conservative difference scheme. II. Monotonicity and conservation combined in a second-order scheme," Journal of Computational Physics, Vol. 14, No. 4, 1974, pp. 361-370.

${ }^{21}$ Bultelle, M., Grassin, M., and Serre, D., "Unstable Godunov discrete profiles for steady shock waves," SIAM Journal on Numerical Analysis, Vol. 35, No. 6, 1998, pp. 2272-2297.

${ }^{22}$ Noh, W. F., "Errors for calculations of strong shocks using an artificial viscosity and an artificial heat flux," Journal of Computational Physics, Vol. 72, No. 1, 1987, pp. 78-120.

${ }^{23} \mathrm{Shu}$, C.-W. and Osher, S., "Efficient implementation of essentially non-oscillatory shock-capturing schemes, II," Journal of Computational Physics, Vol. 83, 1989, pp. 32-78. 